\title{
Entrepreneurship in the Laboratory Can Save Lives
}

\section{Phillip Phan (Johns Hopkins University)}

\section{KEYWORDS: Health Care \& Biotech, Entrepreneurship, Innovation, Management, Marketing, Strategy, Technology Commercialization.}

"Entrepreneurship starts with the discovery of an economic opportunity, which is preceded by a conjecture of the relative value of resources" (Shane and Venkataraman, 2000)

The above quote could easily say, "Saving lives starts with a biomedical discovery embedded in an economic opportunity, which is preceded by a conjecture of the relative value of resources."

We know that scientific discoveries are happening all the time in research laboratories around the world. At Johns Hopkins, a $\$ 2$ billion academic research enterprise, biomedical discoveries are made literally every day. However, relatively few of these discoveries make it to the bedside as lifesaving therapies, devices and other clinical interventions. Perhaps this is the nature of biomedical discoveries: one has to tap many dry wells to find a gusher.

We know that the high failure rate of drugs in development occurs at phase 2 (is it effective?) and phase 3 (is it better than current therapies?) clinical trials. However, I wonder if the problem has a simpler explanation: the discovery's commercial viability isn't being considered early enough, dooming it to failure. Conversely, considering the commercial potential early on can improve the chances that the breakthrough will reach the marketplace.

\section{Shift the Emphasis}

In recent years, the National Institutes of Health have greatly emphasized translational research in funding priorities. Translational research refers to the additional development work needed to derive a proof of concept, which can be tested in animal models of increasing complexity before human trials. However, translation, as it has been practiced in medical centers, has fallen short of its promise. Here is why: it has focused too narrowly on basic research processes and stops at the transition from research to development. In development work, the influence of the marketplace becomes a major factor in determining the shape of the proof of concept.

We know that an innovation in healthcare can help patients only if it makes it to market. While many factors influence whether this happens, a big one is whether insurance companies and other payers will cover the intervention. The basis for a reimbursement is the cost effectiveness and/or marginal increase in efficacy of the intervention. Consider that bioengineers at Johns Hopkins developed a non-toxic, bioreactive adhesive that could be used to repair cartilage in orthopedic patients. However, market research by a team of MBA students showed that insurers and other payers would be more willing to cover the cost of the breakthrough if it were used in eye surgery. This changed the course of the research.

\section{Make it Scalable}

Another factor that drives early market adoption is the medical evidence to support use. The adoption of new therapies and procedures is based on a standard of care supported by evidence. At Johns Hopkins -- a global leader in research on personalized medicine or treatment customized for the single individual -this means that researchers have to prove their technologies can be applied to a large population. When a Johns Hopkins team of computer bioengineers developed a computer-aided system to detect tumors in diseased tissue, they worked with an MBA team, and found that stakeholders needed more evidence before they were willing to adopt the technology. So the researchers published more scientific papers explaining the method and sought input from stakeholders more actively during the preclinical development stage.

\section{Consider the Market Early}

These examples show that it is important for the inventor to seek market information during the design process by exposing the idea to potential payers, users 
and regulators for feedback. Such feedback, in turn, influences the design and/or development efforts to ensure that the needs of both patient and providers are fully met. Without this dynamic process, what may appear to be a scientifically advanced discovery may fail to see the light of day.

In the best circumstances, the combination of entrepreneurial drive, inventor creativity and accurate market intelligence will ensure that the best discoveries make it to market in the quickest possible way. The way to achieve this is to manage biomedical innovation with trans-disciplinary teams of scientists, marketers and entrepreneurs working with one another in a constant conversation. New drugs and therapies developed this way will move iteratively into and out of the laboratory as the design improves, matures and moves closer to proof of concept, testing, trials and launch. In this sense, entrepreneurship saves lives -- because without the skills and market insights of the entrepreneur, lifesaving discoveries in the laboratory will stand little chance of showing up at the patient's bedside. 